\title{
L'immigration et ses espaces
}

\author{
Géographie des populations d'origine étrangère à Lausanne
}

\begin{abstract}
Résumé
Cet article étudie la répartition spatiale des personnes d'origine étrangère dans l'agglomération lausannoise. A l'aide des méthodes traditionnelles de l'écologie factorielle urbaine, il tente de répondre à la question de l'éventuelle ségrégation spatiale de ces populations selon les nationalités. En conclusion, il met en évidence les limites de la méthode utilisée et la nécessité d'une prise en compte du caractère spatialement discontinu et socialement construit des phénomènes d'intégration et de ségrégation.
\end{abstract}

\section{Zusammenfassung}

Dieser Beitrag untersucht die räumliche Verteilung der ausländischen Bevölkerung in der Agglomeration Lausanne. Mit Hilfe der traditionellen Methoden der Faktorökologie versucht man die Frage der räumlichen Segregation dieser Bevölkerungsgruppen zu erfassen.

Als Ergebnis werden die Grenzen der angewandten Methode aufgezeigt und auf die Notwendigkeit hingewiesen, nicht nur auf der räumlichen, sondern auch auf der soziologischen Ebene den Integrations- und Segregationsphänomenen nachzugehen, die sich in einem ständigen Wandlungsprozeß befinden.

Avec $31 \%$ de résidents d'origine étrangère, Lausanne est, derrière Genève (42\%), la grande ville de Suisse ${ }^{1}$ qui compte la plus forte proportion d'étrangers. ${ }^{2} \mathrm{Ce}$ chiffre, élevé en comparaison internationale, représente près du double de la présence étrangère moyenne en Suisse ( $18 \%)$. Il est proche de celui que connaissait la ville de Chicago dans les années 20-30 à l'époque des premiers travaux de sociologie urbaine sur la répartition spatiale des étrangers (JOSEPH 1993). Dans l'espace lausannois, ces quelques 40000 personnes ne se répartissent pas de manière uniforme. Choix et contraintes de la localisation résidentielle font émerger, selon les quartiers, de plus ou moins fortes concentrations qui font de l'espace lausannois à la fois le reflet et l'acteur des processus d'intégration, d'assimilation ou de ségrégation des populations. Dans le cadre d'une réflexion prospective sur la Suisse de demain, la question des migrations est centrale. Épargnée jusqu'ici par les phénomènes qui, dans les pays voisins, font de la question des banlieues, de l'immigration et de la gestion urbaine un sujet brûlant (BOUMAZA 1989; ROMAN 1991, 1993), la Suisse se doit cependant d'examiner sa politique migratoire dans une perspective à long terme qui ne soit plus axée sur la seule dimension économique. La prochaine mise sur pied d'un programme national de recherche "migrations et relations interculturelles" témoigne d'une prise de conscience accrue de ces questions. L'expression spatiale des phénomènes migratoires apparaît comme essentielle à une telle réflexion tant il est vrai que la ségrégation économique ou sociale, qui est à la source du "problème de l'immigration" dans les pays voisins, se double souvent d'une ségrégation spatiale de certains groupes d'étrangers et débouche parfois sur une véritable relégation (DELARUE 1991). Cet article a pour but d'offrir, à partir du cas de Lausanne, un premier aperçu de la répartition spatiale des communautés étrangères et de poser ainsi les prémisses d'une recherche dans ce domaine.

Peut-on lire dans l'espace les marques de l'intégration ou de la ségrégation des populations? Y a-t-il homogénéité dans la répartition territoriale des étrangers? Peut-on au contraire parler de quartiers immigrés, voire de quartiers ethniques? Y a-t-il confinement de certaines populations dans certains types de quartiers? A quels facteurs les différentes localisations peuvent-elles être rattachées? Telles sont certaines des questions auxquelles il s'agit de répondre. De nombreux travaux s'y sont déjà attachés dans plusieurs villes européennes. On peut en tirer trois conclusions majeures (BATTEGAY 1992):

1. Il n'y a pas de ghetto en Europe et la ségrégation n'y est d'une manière générale pas comparable à celle des villes américaines.

2. On peut distinguer deux grands facteurs de ségrégation spatiale, l'un a trait aux catégories sociales, l'autre à la nationalité. La ségrégation des populations selon la nationalité ou l'ethnie est souvent plus forte que la ségrégation selon les catégories sociales et elle agit de manière différente.

3. La déconcentration spatiale n'est pas l'unique aboutissement de l'intégration historique des minorités. Une cohorte d’immigrants ne se dissout pas nécessairement à travers la ville au fil du temps.

En Suisse, les travaux de RAFFESTIN (1982 et 1984) rejoignent la première de ces conclusions: jusqu'en 1980, les grandes villes suisses sont caractérisées par des différences faibles entre les quartiers en matière de concentra-

Etienne Piguet, maître assistant à l'Institut de Géographie de I'Université de Lausanne, assistant dipl. à l'Ecole des HEC, BFSH 2, 1015 Lausanne-Dorigny 
tions étrangères et on ne note aucun phénomène de ségrégation.

L'évolution récente des migrations vers la Suisse, caractérisée par l'arrivée importante de migrants en provenance de nouveaux pays (Portugal, Afrique, Asie), et par la stabilisation des populations étrangères, que traduit l'augmentation constante du nombre des permis de résidence ( C ») par rapport aux permis de séjours («A» et «B»), justifie cependant que ces conclusions soient remises à jour et évaluées en fonction de l'origine des différentes populations. Avant d'entamer une telle approche, il semble nécessaire de prendre quelques précautions concernant le statut des concepts et des catégories auxquels nous avons recours.

\section{Des catégories ambiguës}

C'est dans le champ d'étude des relations inter-ethniques que règne l'un des plus grand flou en matière de définition et d'usage des concepts (SIMON 1993). La catégorie binaire étranger/national est purement juridique, elle est certes attestée puisqu'elle fait référence à une inscription administrative, mais reste totalement extérieure à la personne elle-même. ${ }^{3}$ Telle que nous l'avons utilisée jusqu'ici, elle apparaît donc comme largement artificielle et son usage doit être légitimé. Cet usage se justifie ici dans la mesure où on envisage les catégories nationales comme des indices et où on fait l'hypothèse qu'elles recouvrent ou recoupent en partie des caractéristiques effectives de certains sous-groupes de la population. Ces caractéristiques sont difficilement identifiables a priori: une éventuelle ségrégation, le fait qu'existent des localisations urbaines spécifiques, l'intensité des liens sociaux entre personnes de même origine, l'existence de pratiques de l'espace spécifiques à certaines groupes, etc... Elles ne sont pas nécessairement partagées par tous les membres d'un groupe national, mais l'utilisation de la catégorie nationale, aisément saisissable parce que juridique, établie et recensée, permet de disposer d'un «marqueur» qui en permet l'approche. Une telle démarche ne représente qu'une première étape qui, si elle ouvre certaines voies de recherche, devra être complétée pour arriver véritablement à comprendre le sens des phénomènes dont les résultats statistiques ne sont que la trace. On rejoindra ainsi une géographie des communautés étrangères plus interprétative (LEY 1988), qui ne fera pas l'objet de ce travail mais en est un prolongement nécessaire dont on esquissera le profil.

Ces quelques précautions prises, nous pouvons maintenant envisager une première approche des dimensions spatiales de la présence étrangère, d'abord à l'échelle de l'agglomération, puis à celle des sous-secteurs statistiques de la ville de Lausanne.

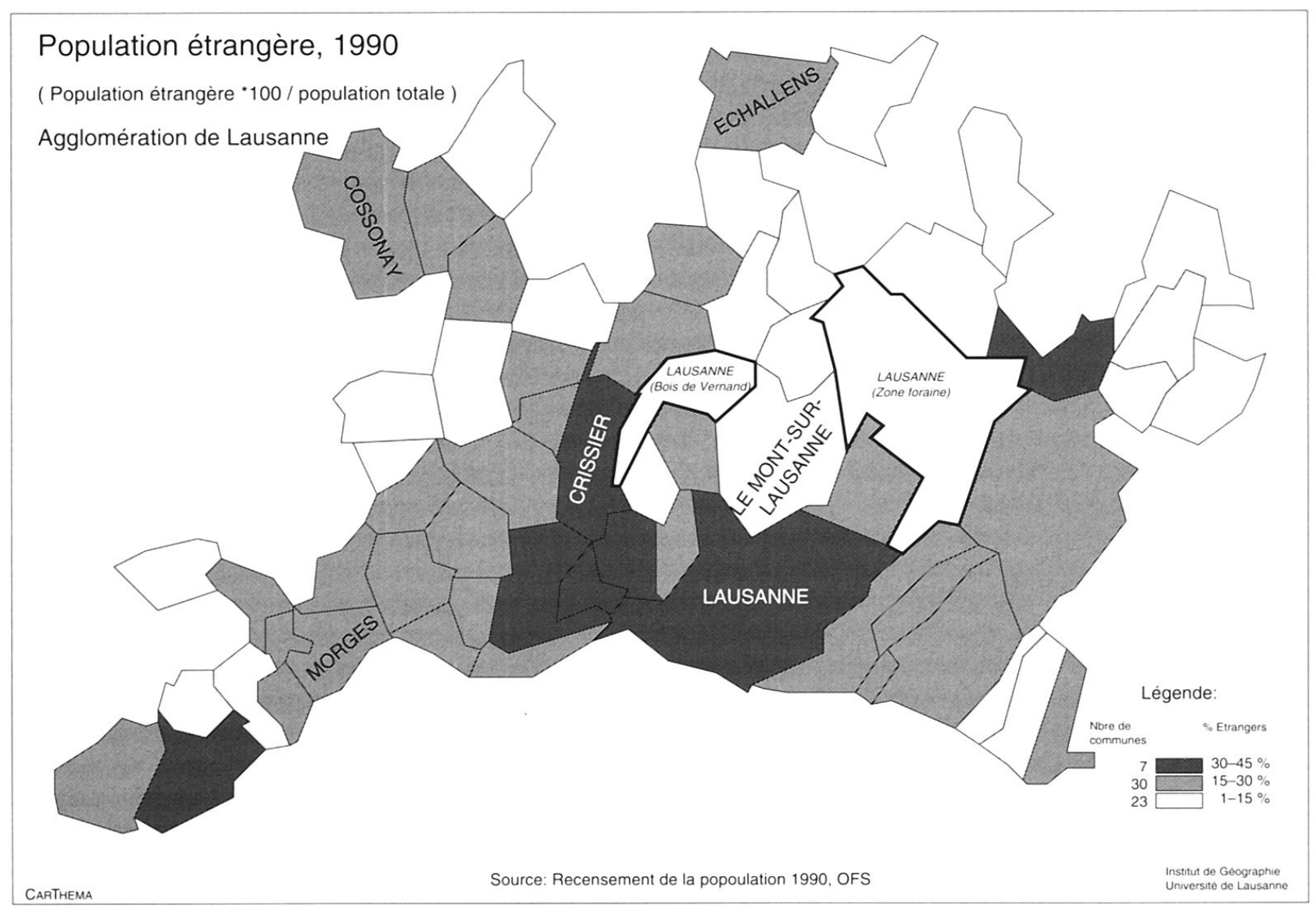

\section{Carte 1}




\section{Les étrangers dans l'agglomération de Lausanne}

Les soixante communes de l'agglomération lausannoise rassemblent une population totale d'environ 300000 personnes dont 82000 étrangers. On constate d'importantes différences selon les communes dans le poids de ces communautés étrangères (carte 1$){ }^{4}$

Une nette répartition centre-périphérie apparaît sur cette carte, c'est dans les communes du centre et du centre-ouest de Lausanne: Prilly, Renens, Chavannes et Crissier, que se trouvent les plus fortes proportions d'étrangers. Les communes de Renens et Crissier avec chacune $44 \%$ d'étrangers dépassent de loin la ville centre. Ce sont les deux communes de Suisse oú la présence étrangère est la plus forte. Les centres secondaires de Morges, Echallens et Cossonay apparaissent eux aussi avec des proportions d'étrangers significatives. ${ }^{5}$ A l'inverse, les communes rurales comme Servion ou Mézières comptent moins de $5 \%$ de résidents étrangers.

Cette premiere carte souligne le caractère urbain de la présence étrangère. En 1889, RAVENSTEIN avait déjà énoncé cette propriété dans ses "lois de la migration". Plusieurs facteurs semblent jouer un rôle ici, tous rattachés aux principes de diversité et de mouvement qui caractérisent la ville.

- La rotation plus rapide des emplois est favorable à l'insertion des migrants sur le marché du travail; la rotation des habitants leur permet de se loger sans trop de difficultés.

- L’intensité des interactions propre à la ville donne accès à une quantité d'informations dont le migrant est par nature demandeur; elle permet une connexion facilitée à une multitude de réseaux parallèles formels ou informels (associations, clubs, amis, etc...) qui permettent l'insertion du nouvel arrivant.

- Certaines des fonctions économiques de la ville caractérisées par une dualisation particulièrement marquée du marché du travail nécessitent la présence massive de migrants dont les qualifications et la précarité répondent aux besoins d'un tertiaire banal qui regroupe des activités de nettoyage, de services personnels, de commerce, etc... (PIORE 1979).

- En regard de la tendance actuelle à la suburbanisation et à l'accroissement de la pendularité, certains groupes de migrants se trouvent peut-être placés dans une position défavorable en matière de mobilité et de possibilités de logements qui les contraint à une localisation plus proche des centres où se situent leurs lieux de travail.

L'étranger dans la ville apparait donc, parfois au prix de son exploitation économique, comme un élément du cercle vertueux qui permet à la diversité économique, sociale et culturelle de se perpétuer. Le caractère général de la catégorie "étrangers» rend cependant difficile d'interpréter plus avant ces localisations. Il semble évident qu'une distinction entre pays d'origine est nécessaire, elle permettra de poser la question des facteurs communs qui pourraient influencer la localisation des ressortissants de certains pays dans l'agglomération. ${ }^{6}$ Une analyse facto- rielle en composantes principales ${ }^{7}$ effectuée sur la base des pourcentages des différentes nationalités présentes dans les communes permet de mettre en évidence deux regroupements majeurs: Un premier groupe de personnes originaires d'Espagne, Italie, Asie, Amérique du Sud, Afrique et Ex-Yougoslavie se localise selon une première logique (carte 2). C'est à ce premier groupe que s'applique l'idée de centralité. C'est aussi celui qui semble se localiser le plus nettement en fonction de la disponibilité en logements bon marchés, la corrélation entre le poids factoriel des communes et le niveau moyen des loyers $^{8}$ est très significativement négative $(r=-0.5)$.

Un deuxième groupe, formé des personnes originaires d'Europe du Nord, d'Allemagne, de France et d'Amérique du Nord, se localise de manière très différente (carte 3 ). Toujours absent de la périphérie campagnarde lointaine, mais aussi des centres urbains, ce groupe favorise la bordure immédiate des deux principaux centres: la première couronne morgienne (Tolochenaz, Chigny, Echichens, Préverenges, St-Sulpice) et l'est lausannois (Pully, Paudex, Lutry, Belmont, Savigny, Epalinges) ainsi que les communes isolées de Jouxtens-Mézery et de Mex.

Les communes fortement marquées par la présence de l'un ou l'autre des deux groupes different fortement par leur statut socio-économique et leur niveau moyen de revenu par habitant. Les membres du groupe 2 se localisent préférentiellement dans les communes "riches» (plus de $20 \%$ d'habitants avec un revenu supérieur à 75000 francs/an en 1990); ceux du groupe 1 dans les communes "pauvres» (plus de $25 \%$ d'habitants avec un revenu inférieur à 29900 francs/an). ${ }^{9}$ On remarque en outre que les taux d'impositions, ${ }^{10}$ qui varient entre les communes de l'agglomération de 50 à $120 \%$ de l'impôt cantonal de base, sont particulièrement bas dans les communes à forte présence étrangère du groupe 2 (les communes à plus de 0.5 écarts types ont toutes des taux inférieurs à $90 \%$ ). La présence des résidents du groupe 2 dans les communes isolees de Mex (75\% de taux d'imposition) et de Jouxtens $(80 \%)$ semble bien relever d'un tel "choix fiscal».

On peut dire en résumé que si l'un des groupes a le pouvoir de choisir véritablement sa localisation en fonction de parametres fiscaux et de standing, l'autre la subit en étant contraint à chercher des loyers inférieurs et des logements disponibles dans des communes où les taux d'imposition sont souvent plus élevés. Il y a bien ici une forme de ségrégation, mais elle ne s'opère pas seulement entre Suisses et étrangers et recouvre un phénomène socio-économique plus large, qui touche aussi bien certaines couches de la population suisse. Cette coïncidence de plusieurs facteurs ségrégatifs tend à relativiser, à cette échelle, la conclusion relevée plus haut à l'échelle européenne, selon laquelle la ségrégation ethnique des populations est plus forte et agit de manière différente de celle des catégories sociales. Entre les communes de l'agglomération lausannoise, de larges recoupements existent entre les facteurs de ségrégation spatiale liés à la nationalité et ceux liés à la dimension économique. 


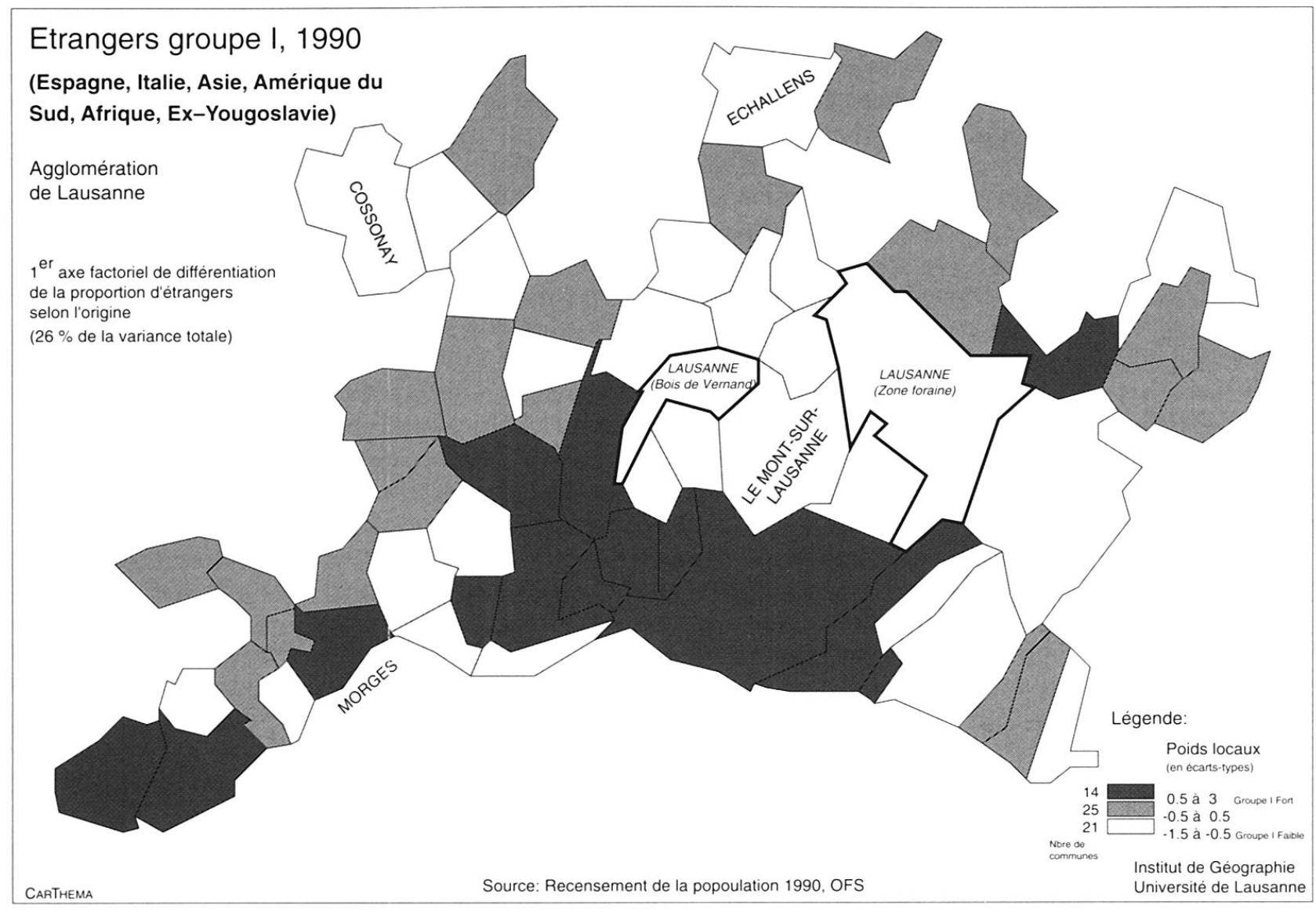

\section{Carte 2}

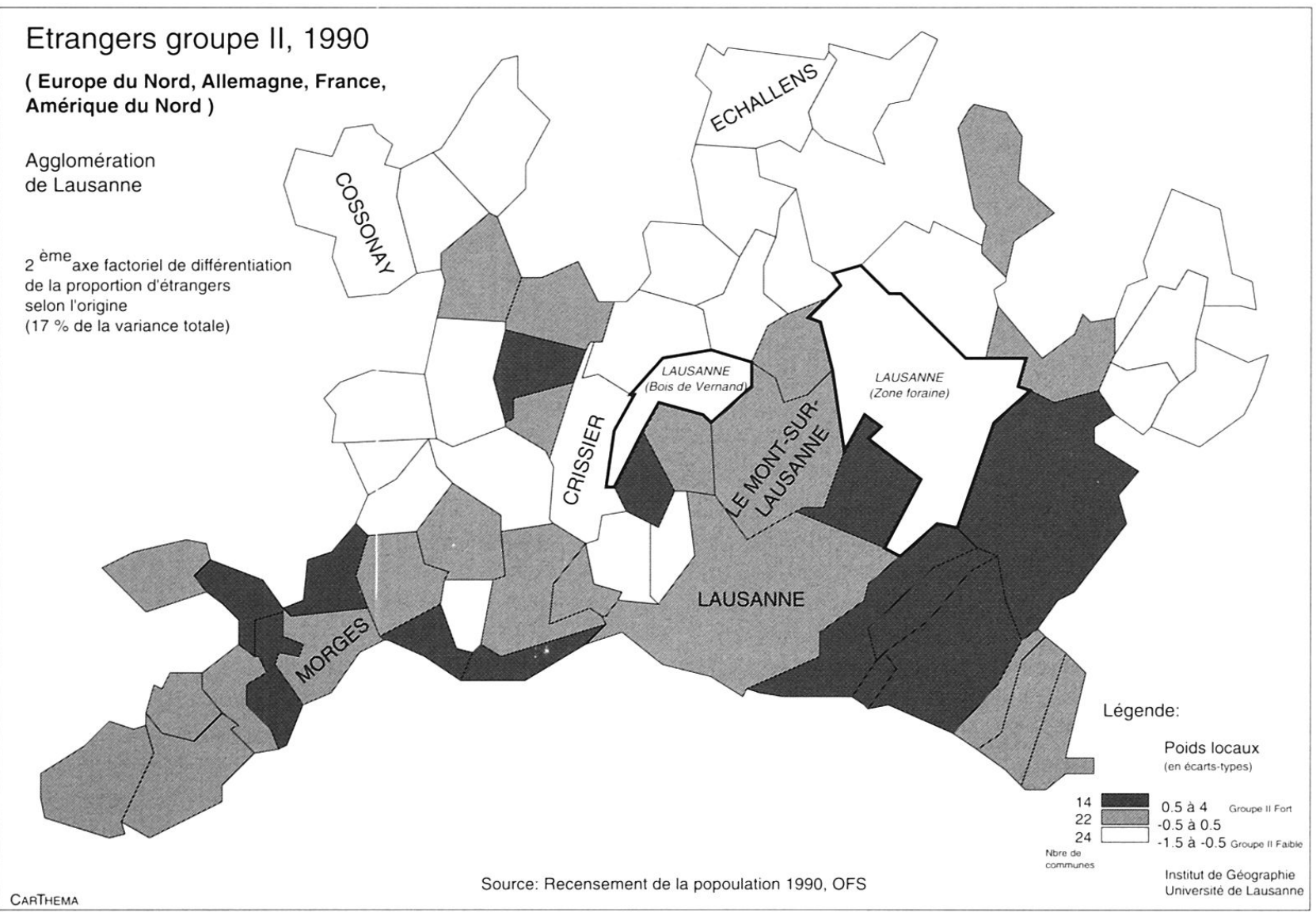

\section{Carte 3}


Une comparaison basée sur un indice simple de dissimilarité $^{11}$ (VANT 1983), qui indique à quel point les populations sont concentrées dans un seul espace (ID = 1) ou réparties de manière homogène (ID $=0$ ), confirme cette impression:
ID populations étrangères
$=0.15$
ID revenus 1990 supérieurs à $75000 \quad=0.20$
ID revenus 1990 inférieurs à $29900=0.10$

Certaines populations sont plus fortement concentrées: Amérique latine (0.24), Afrique (0.22), Espagne (0.21), Asie (0.19), et d'autres moins: Portugal (0.14), Allemagne (0.11), France (0.9). Même si on peut relever un lien apparent entre une hypothétique "distance culturelle" propre à certains groupes et des concentrations spatiales plus marquées, on constate que les disparités restent néanmoins faibles. Même pour les plus fortes concentrations, il suffirait de peu de mouvements pour arriver à une homogénéité totale. Si des tendances aux regroupements se dessinent, les pourcentages d'explication liés aux facteurs restent faibles. La plupart des communes de l'agglomération comptent en fait parmi leurs habitants des représentants de toutes les origines. Si le poids de chaque groupe varie, il se double aussi d'une remarquable mixité.
La description de la présence étrangère à l'échelle de l'agglomération fournit une première série de résultats. Il s'agit maintenant de les comparer à ceux obtenus à l'échelle de la commune de Lausanne afin de voir si des logiques similaires de répartition et de concentration se retrouvent.

Dans la carte qui suit, on a représenté la proportion d'étrangers dans les 81 sous-secteurs statistiques de la ville (carte 4$){ }^{12}$

La répartition centre-périphérie est moins marquée sur cette carte, où émergent cependant de nettes disparités: La Bourdonnette ( $50 \%$ de population étrangère) - Malley - Prélaz (46\%) - Route de Morges (52\%) - Av. d'Echallens - Maupas et Chauderon sur une ligne ouest-est, Riponne-tunnel (49\%) - La Borde - La Cité - Le Vallon et Marterey sur un axe nord-sud et enfin Ouchy regroupent les plus fortes concentrations d'étrangers. Ici aussi pour comprendre les choses, il est important de faire une distinction par nationalité. Une analyse factorielle du même type que celle déjà présentée donne des résultats très proches (carte 5).

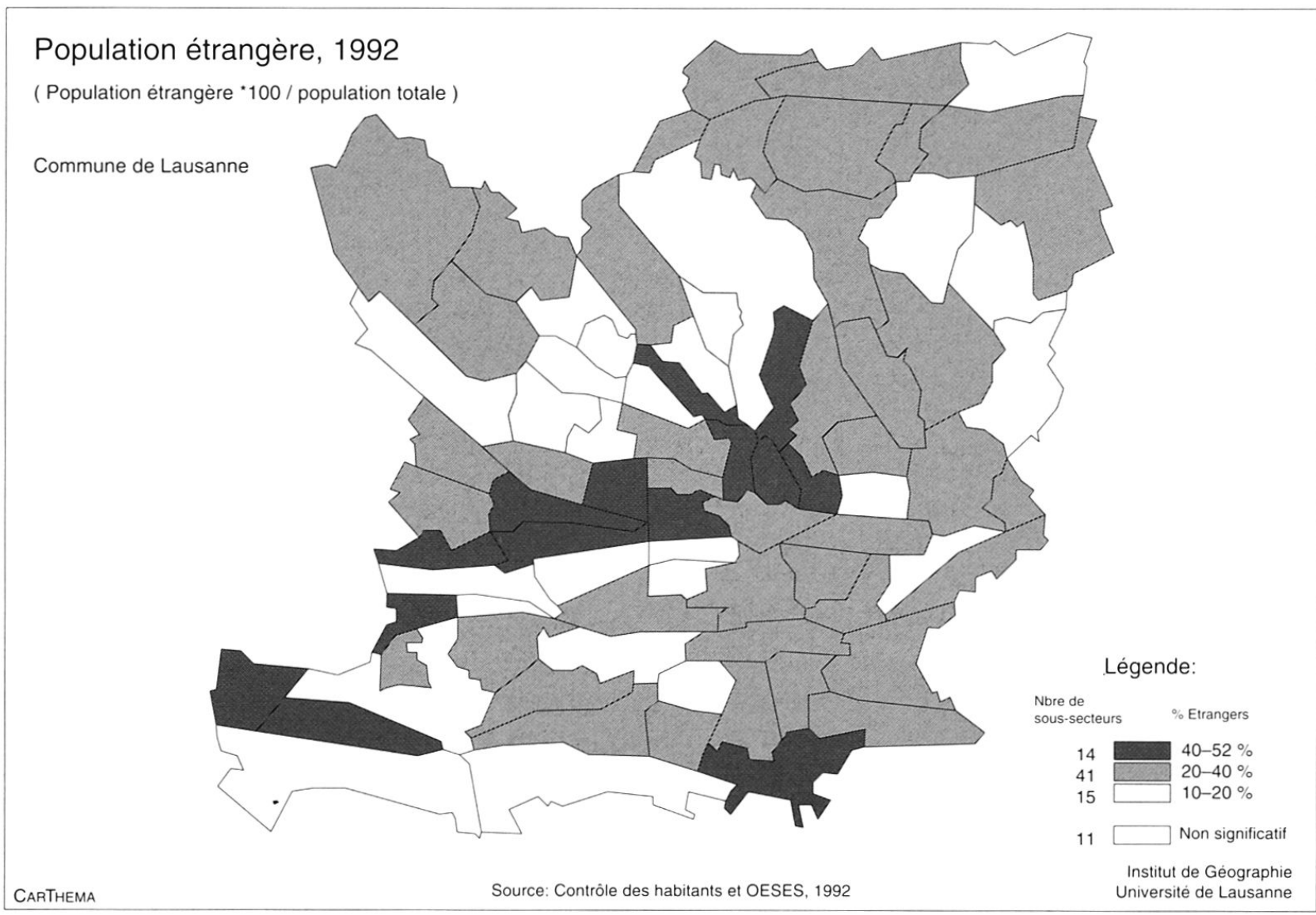

Carte 4 


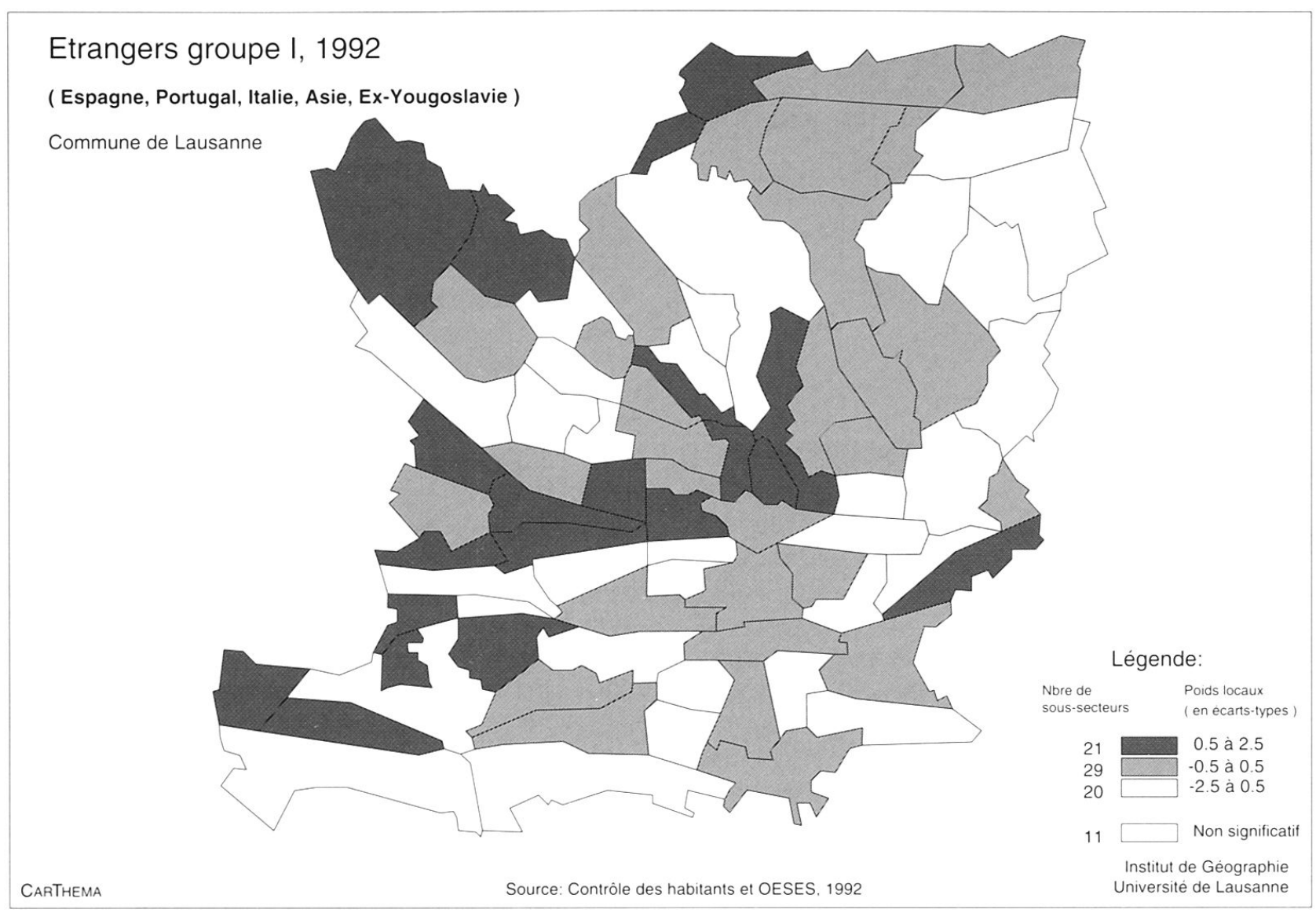

\section{Carte 5}

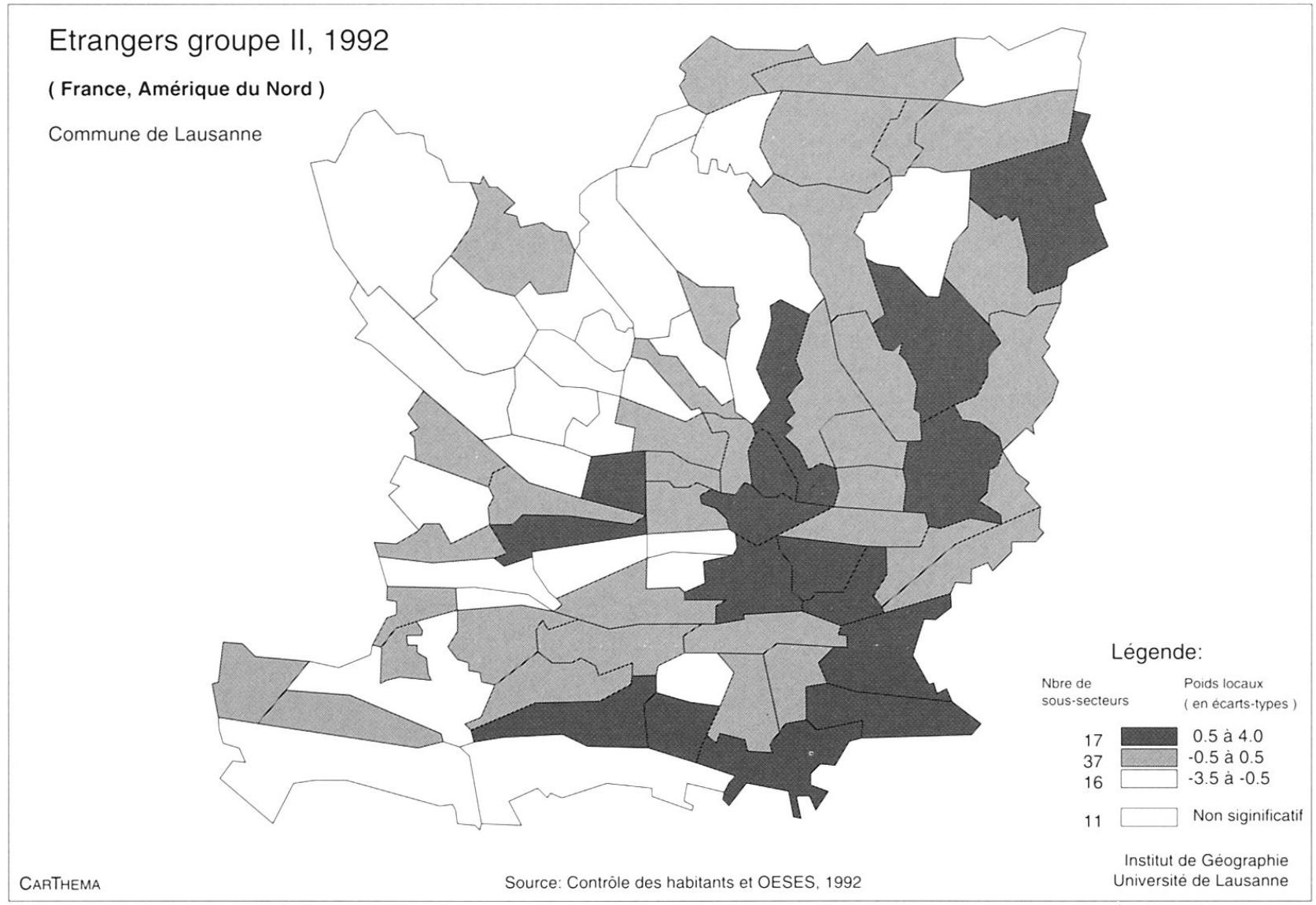

Carte 6 
Un premier groupe formé du même type de populations qu'à l'échelle de l'agglomération se localise préférentiellement dans des quartiers qui offrent des logements bon marché, surtout dans des immeubles anciens (Prélaz R. de Morges - Av. d'Echallens - Maupas - Chauderon et toute la zone autour de la Cité) ou dans des zones plus récentes comportant des grands immeubles locatifs (Bourdonnette, Blécherette, Chablière). La corrélation entre les poids factoriels sur ce premier facteur et le loyer moyen est très significativement négative $(r=-0.5)$.

Le deuxième groupe dont la composition coïncide aussi avec les résultats de l'agglomération se localise clairement à l'est de Lausanne, Cour - EPFL - Elysée - Montchoisi - Florimont et Georgette - Gare/Petit-chêne mais aussi du côté de la Cité, du Vallon et de Marterey oú les deux groupes se rencontrent. On trouve aussi des personnes du groupe 2 dans les quartiers résidentiels de Chailly - Av. Secrétan. Dans tous ces quartiers, on peut lire la marque d'une concentration en fonction du niveau socio-économique. Elle semble cependant moins marquée qu'à l'échelle de l'agglomération (carte 6).

L'indice de dissimilarité donne les résultats suivants: pour l'ensemble de la population étrangère ID $=0.18$, Afrique (0.25), Espagne (0.25), Asie (0.25), Portugal (0.24), Italie (0.15), France (0.14). Même s’ils ne sont pas directement comparables à deux échelles différentes (woODs 1976), les indices de la ville et de l'agglomération confirment bien l'impression générale de concentrations liées au statut. Il n'est cependant pas possible de dire sans investigations supplémentaires si les différences entre les nationalités se rapportent à des variables strictement économiques ou a des phénomènes de regroupement, volontaires ou involontaires. La part d'une ségrégation purement ethnique, dont on a vu en introduction qu'elle joue un rôle important dans d'autres pays, reste en tout état de cause faible à Lausanne.

A l'issue de cette description de l'espace lausannois, nous pouvons maintenant revenir à nos questions de départ. Notre premier constat est nuancé: il n'y a pas homogénéité et il existe bien des quartiers à très forte population étrangère. On ne saurait affirmer pour autant qu'il y a confinement de certaines populations dans certains types de quartiers en raison même de leur nationalité. En ce sens, on peut clairement rejeter l'hypothèse du ghetto. Certaines populations apparaissent avec des localisations plus marquées que d'autres. Issues surtout de vagues migratoires récentes, elles laissent à penser que la diffusion spatiale peut dans une certaine mesure être considérée comme un indicateur de la plus ou moins grande assimilation des populations. Il faut cependant rappeler avec RAFFESTIN (1984, p. 94) que "rien n'autorise à inférer que les indices de concentration faibles et forts signifient respectivement non-discrimination et discrimination. La non-discrimination géographique peut cacher une discrimination insidieuse dans les rapports quotidiens et la discrimination géographique ne s'accompagne pas ipso facto d'une discrimination dans les rapports quotidiens.» Nos conclusions s'avèrent en fin de compte optimistes, puisque la ségrégation spatiale en fonction des nationalités est faible et qu'elle recouvre dans une large mesure une ségrégation économique qui, peut-être à tort, paraît moins choquante. Il serait cependant prématuré d'en tirer pour l'avenir la conclusion que le non-interventionnisme politique, qui a été de règle au niveau suisse en matière de populations étrangères, débouche sur un «modèle suisse» en matière d'intégration. D'autres travaux (BOLZMANN, FIBBI \& VALENTE 1992) ont montré que «la position statutaire des immigrés de longue date en Suisse est caractérisée par la participation socio-économique et l'exclusion politique», ce qui ne va pas sans frustrations. Les développements actuels de l'immigration, la fin prochaine de la rotation de main-d'œuvre saisonnière et du système des Gastarbeiter qui tentaient en fin de compte de dissocier les besoins du marché du travail de la question de l'intégration, donnent à nos conclusions un caractère provisoire: rien ne dit que la Suisse de demain pourra continuer, avec une population étrangère aussi importante, à éviter que la question des étrangers ne prenne plus d'ampleur.

Les méthodes que nous avons employées jusqu'ici pour rendre compte de l'espace des migrations sont loin d'en avoir saisi toutes les dimensions. C'est peut-être là aussi que doit être cherchée la cause d'un optimisme peut-être excessif. Nos analyses souffrent d'une faiblesse majeure en ce qu'elles appréhendent l'espace avec l'idée d'une continuité des phénomènes qui amène à assimiler la ségrégation spatiale à l'inexistence de mélanges et de voisinages. Cette approche nous a certes permis d'obtenir certains résultats et elle apparaît comme une première étape nécessaire, mais elle doit être complétée par une appréhension du caractère potentiellement discontinu de l'espace migratoire. Nous voudrions brièvement évoquer cette voie de recherche en guise de conclusion et en tant qu'ouverture sur des travaux futurs.

\section{Réseaux de relations et production sociale de l'altérité}

C'est la participation des individus à une multitude de réseaux qui définit l'intensité et la forme du lien social. A cet égard, l'existence de réseaux propres à certains groupes issus de la migration peut être vue comme une marque de différentiation voire de ségrégation au même titre que le lieu de résidence. Cette approche n'est pas pour autant aspatiale, dans la mesure où l'intensité des réseaux génère des phénomènes de mouvements et de circulation, qui marquent et sont marqués dans l'espace et qui représentent des enjeux majeurs en matière de cohabitation harmonieuse des populations à l'échelle urbaine. A Lausanne un commerce d'alimentation sur deux est géré par une personne d'origine étrangère et propose des produits spécifiques (PIGUET 1994). Pour exercer leur activité, ces commerces s'appuient sur d'importants réseaux de clientèle et d'approvisionnement, dont le rayon d'action dépasse de loin la seule agglomération. 
Ils mettent en contact des échelles multiples, qui vont du quartier auquel ils donnent parfois une ambiance bien particulière (rue de Fribourg à Genève, avenue du Simplon à Lausanne), aux relations commerciales internationales avec le pays d'origine. A Lausanne comme ailleurs, ces phénomènes ne sont pas nécessairement liés à une présence simultanée d'étrangers résidents. Ce constat de non-recouvrement entre le marquage ethnique d'un quartier et sa population résidente est important. A Paris, le Triangle de Choisy, appelé souvent la Petite Asie, ne compte que $20 \%$ de population asiatique ce qui conduit Alain BATTEGAY (1992) à dire que "ce type de fonctionnement dessine une configuration de territoires ethniques qui ne s'inscrivent plus dans le paradigme du village urbain: leur composition résidentielle hétérogène, leur fonctionnement interne fragmenté les éloignent d'un espace d'interconnaissance, et leur tissu commercial et économique leur donne des fonctions de centralité qui s'ancrent localement mais qui dans le même mouvement lient le quartier à la ville ou à l'agglomération, et en spécifient les fonctions urbaines». Un autre exemple de telles logiques discontinues peut être trouvé dans le fonctionnement des très nombreuses associations nationales ou régionales qui regroupent les étrangers à Lausanne (FIBBI 1993). Là aussi, le réseau génère des configurations spatiales qui demandent à être étudiées comme des «conjonctions de deux logiques, une logique de l'espace résidentiel et des processus de territorialisation aboutissant à des aires naturelles localisées et marquées sur le plan morphologique, et une logique de l'espace public, et du procès de communication entre des territoires définis comme des régions de signification délocalisées et fragmentées" (JOSEPH 1984). Afin d'être suivie, cette voie de recherche implique la prise en compte du fait qu'un quartier ne devient ethnique qu'au moment où il est ressenti comme tel par certains groupes de population, habitante ou non. Bien plus que des indices quantitatifs, c'est la prise en compte du sens donné par les acteurs aux phénomènes auxquels ils sont parties prenantes et du fait que, loin d'être des «données du problème», l'ethnicité, l'intégration ou la ségrégation sont constamment recréées, qui permettra de comprendre la relation entre allogénéité des populations et espace (SMITH 1992). Ce travail à l'échelle suisse pourra largement s'inspirer de l'important travail réalisé dans les pays anglo-saxons sous l'égide de la géographie humaniste (LEY, CLARKE \& PEACH 1984).

\section{Conclusion}

C'est un modèle en pleine transition auquel la Suisse est confrontée en matière de présence étrangère. Même si la conjoncture économique actuelle freine temporairement ce processus, la Suisse de demain continuera immanquablement à connaître un accroissement de la diversité de sa population. Si à l'heure actuelle on a pu montrer que sur le plan spatial les phénomènes de ségrégation restent faibles, il n'en demeure pas moins qu'une réflexion de fond sur la question de l'intégration est nécessaire. Cette réflexion devra tenir compte du fait qu'à l'heure où l'échelle du quartier et de l'international se recoupent, où les mouvements circulatoires périodiques s'intensifient, oủ la télévision par satellite permet à chacun de s'informer dans sa propre langue, la dislocation et l'hétérogénéité deviennent la règle. Il s'agit dès lors de trouver les méthodes pour rendre compte de ces évolutions. Nous avons esquissé une piste de recherche possible pour nourrir une telle réflexion, le lancement du Programme national 39 sera une première occasion d'aller dans ce sens. Si elle est suivie, la proposition de l'Association Suisse des Sciences Humaines de mettre sur pied un Forum permanent d'étude des migrations en sera l'indispensable complément à long terme. Cet effort de réflexion et de recherche sera le prix à payer pour maintenir dans la Suisse de demain une cohabitation harmonieuse des populations.

\section{Notes}

'Nous considérons comme grandes villes les neuf communes de plus de 50000 habitants.

${ }^{2}$ Données 1990, source: Union des villes suisses et OFS (1993).

${ }^{3}$ Un enfant né en France de parents marocains qui émigre par la suite en Suisse sera Français. Le même, né en Allemagne, sera Marocain. S'il est né et a été élevé en Suisse, il restera Marocain, sauf naturalisation. Enfin si il est né au Maroc et a été élevé au Maroc mais que son grand-père était Suisse il sera Suisse...

${ }^{4}$ Une carte de repérage portant les noms des communes se trouve en annexe. Les cartes qui suivent ont été réalisées à l'aide du Logiciel CARTHEMA (COSINSCHI \& GABIOUD 1994). Je tiens à remercier M. G. Clivaz, cartographe, pour son aide dans la réalisation de ces cartes.

${ }^{5}$ II faut garder en mémoire à la lecture d'une telle carte le fait que dans certaines petites communes (Montpreveyres par exemple avec 302 habitants) la population totale ne permet pas de considérer la proportion d'étrangers comme très significative.

${ }^{6}$ On compte dans l'agglomération, selon le RFP 90, 26\% d'Italiens, $18 \%$ d'Espagnols, 15\% de Portugais, 10\% de Français, 7\% d'Asiatiques, 7\% d'Allemands, 5\% d'Américains, 4\% d'Africains, 4\% d'Ex-Yougoslaves, 4\% d'autres origines.

L'ouvrage de Lena SANDERS (1990) donne une synthèse de l'utilisation de cette méthode en géographie.

${ }^{8}$ Source: OFS, RFP 90

${ }^{9}$ La corrélation entre la proportion de revenus supérieurs à 75000 francs et le poids factoriel sur le facteur 2 est très significative (sign. $\leqq 0.01$ ) avec $r=0.62$. La corrélation entre la proportion de revenus inférieurs à 29900 francs et le poids factoriel sur le facteur 1 est significative (sign. $\leqq 0.05$ ) avec $r=0.32$ (Source: Admin. féd. des contrib.). 
${ }^{10}$ Le taux d'imposition communal est fixé par la commune en proportion de l'impôt cantonal. Selon la richesse du contribuable, l'économie due à une bonne localisation atteint vite plusieurs milliers de francs par an.

"I. D. $=\frac{1}{2} \sum_{\mathrm{i}=1}^{\mathrm{n}}\left|x_{i}-y_{i}\right|$

où $x$ est le pourcentage du groupe urbain $x$ habitant dans la zone i et y le pourcentage du reste de la population urbaine habitant le même quartier. On peut interpréter le résultat comme le pourcentage de la population du groupe $x$ qui devrait déménager pour que la mixité soit parfaite c'est-à-dire pour que le poids du groupe dans chaque quartier soit le même que son poids dans l'ensemble de la ville.

${ }^{12}$ Une carte de repérage portant les noms des sous-secteurs se trouve en annexe. 11 quartiers qui comptent moins de 50 habitants (en blanc sur la carte) on été écartés afin de ne pas fausser l'analyse.

\section{Bibliographie}

BATTEGAY, A. (1992): L'actualité de l'immigration dans les villes françaises: la question des territoires ethniques. Dans: Revue Européenne des Migrations Internationales, vol. 8, no 2, p. 83-97.

BOLZMANN, C., FIBBI, R., VALENTE, L. (1992): Les racines locales des immigrés ou comment inventer une nouvelle citoyenneté. Dans: Espaces et Sociétés, 68, pp. 47-65.

COSINSCHI, M., GABIOUD, B. (1994): Carthema, Logiciel de réalisation automatique de cartes thématiques de I'Institut de Géographie de l'Université de Lausanne. Dans: Travaux et recherches de l'IGUL, no 11.

DELARUE, J.-M. (1991): Banlieues en Difficultés, la Relégation, Rapport au ministre de la Ville. Paris, Syros/Alternatives.

FIBBI, R. (1993): Stratégies identitaires et participation sociale: les racines locales des immigrés. Dans: Les Migrations Internationales, Université de Lausanne, Cours général public 1992-1993, pp. 89-102.
JOSEPH, I. (1984): Situation migratoire et double appartenance culturelle. Dans: KUMPS, A.-M. (dir.): Du bon usage de la ville. Bruxelles, Facultés universitaires Saint-Louis, pp. 95-110.

JOSEPH, I. (1993): Du bon usage de l'Ecole de Chicago. Dans: ROMAN, J.: Ville, exclusion et citoyenneté. Paris, Esprit.

LEY, D., CLARKE C., PEACH, C. (éds.) (1984): Geography and Ethnic Pluralism. Londres, George Allen \& Unwin.

LEY, D. (1988): Interpretative Social Resarch in the Inner City. Dans: EYLES, J. (éd.): Resarch in Human Geography. Oxford, Basil Blackwell, 1988, pp. 121-138.

PIGUET, E. (à paraître), Les commerces étrangers dans l'espace urbain, Actes du colloque de la Société suisse de sociologie, Berne, 22-23 octobre 1993.

PIORE, M. (1979): Birds of passage: migrant labor and industrial societies. Cambridge, Cambridge University Press.

RAFFESTIN, C. (1982): Géographie des groupes nationaux dans les quartiers de la ville de Genève. Dans: CENTLIVRESDEMONT, M. (dir.): Un nouveau regard sur la ville, contribution à l'ethnologie urbaine. Berne, Soc. Suisse d'ethnologie, pp. 139-148

RAFFESTIN, C. (1984): La ville à forte composante étrangère: une mosaïque de territorialités. Dans: KUMPS, A.-M. (dir.): Du bon usage de la ville. Bruxelles, Facultés universitaires SaintLouis, pp. 79-94

RAVENSTEIN, E. (1889): The Laws of Migration. Dans: Journal of the royal statistical society, juin.

ROMAN, J. (dir.) (1993): Ville, exclusion et citoyenneté, Paris, Esprit.

ROMAN, J. (et al.) (1991): Citoyenneté et urbanité, Paris, Esprit.

SANDERS, L. (1990): L'analyse des données appliquée à la géographie. Montpellier, Reclus.

SIMON, P.-J. (dir.) (1993): Vocabulaire historique et critique des relations inter-ethniques. Paris, Pluriel recherches, cahier 1, L'Harmattan.

SMITH, M.P. (1992): Postmodernism, urban ethnography, and the new social space of ethnic identity. In: Theory and Society, 21, pp. 493-531.

UNION DES VILLES SUISSES (1993): Statistiques des villes suisses. Berne, UVS

VANT, A. (1983): Analyse de la ségrégation et géographie sociale. Dans: NOIN, D.: Géographie Sociale, actes du Colloque de Lyon, 14-16 octobre 1982, Paris, Université de Paris I.

WOODS, R. I. (1976): Aspects of the scale problem in the calculation of segregation indices. In: Tijdschrift voor Economische en Sociale Geografie, 1976, pp. 169-174. 
Annexes:
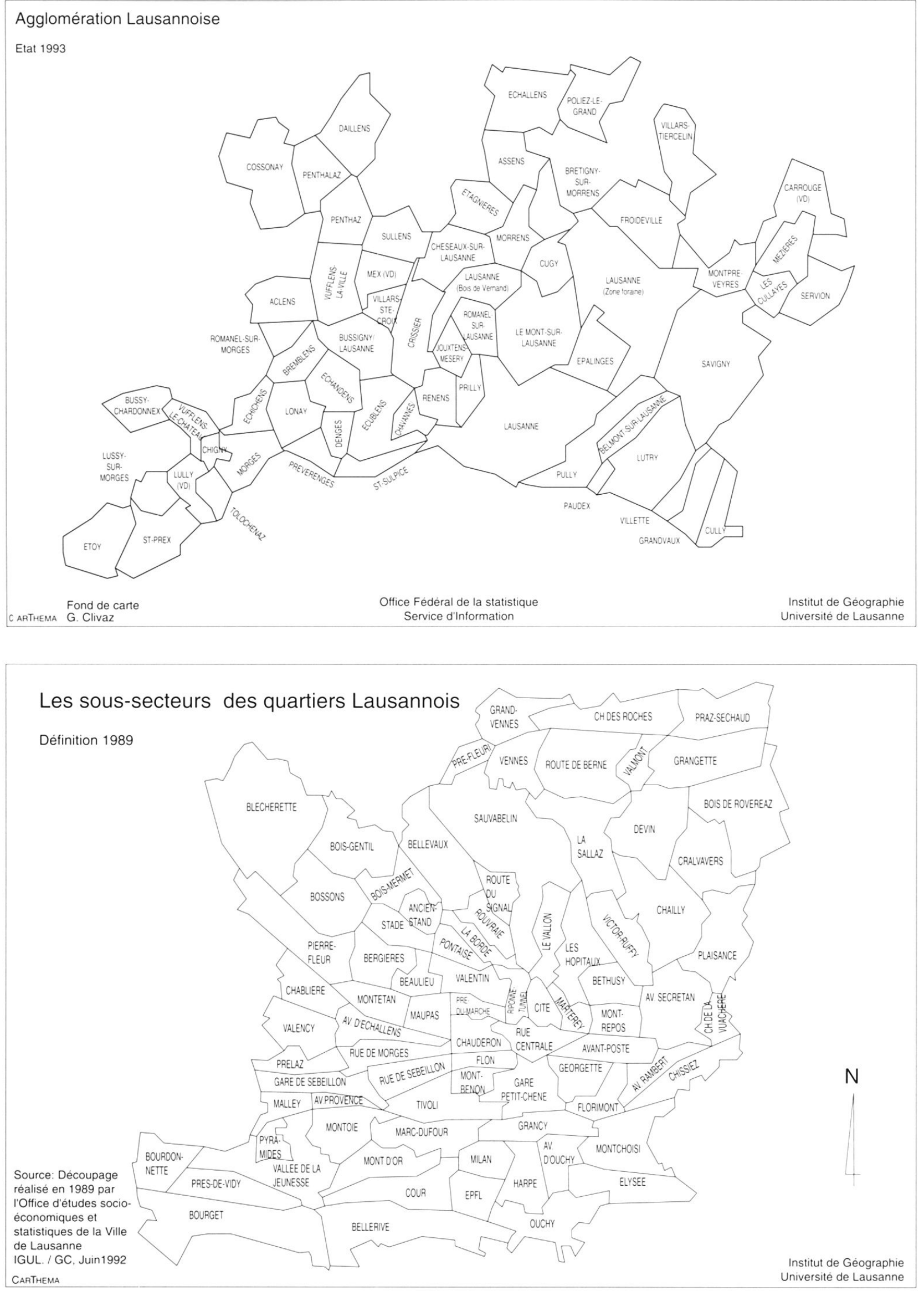